\title{
Nível de atividade física e comportamento sedentário em escolares
}

\author{
Physical activity level and sedentary behavior among students
}

Resumo - Este trabalho objetivou verificar o nível de atividade física (NAF) e o comportamento sedentário em escolares da cidade de Aracaju (SE). Participaram do estudo 1028 estudantes de ambos os sexos, com uma média de 15,38 (2,44) e 15,24 $(2,40)$ anos de idade para o sexo feminino e masculino, respectivamente. Da amostra, $24,7 \%$ eram crianças e $75,3 \%$ adolescentes, com uma média de $12,07(0,88)$ e $16,39(1,72)$ anos de idade, respectivamente. Utilizou-se um instrumento já aplicado em estudos nacionais, com o qual se identificou o tempo médio de horas assistindo TV (hTV) por dia e o NAF - PAQ-C. Para a análise de dados, foram empregados a estatística descritiva, o teste "t" para amostras independentes, o Exato de Fisher e o teste de comparação entre duas proporções. Adotou-se nível de significância de $5 \%(\mathrm{p} \leq 0,05)$. O sexo masculino teve um escore de $2,25(0,60)$ para atividade física, significativamente, maior que o feminino. A prevalência de sedentarismo foi $72,5,89,3$ e 85,2\% para o sexo feminino, considerando os grupos "crianças", adolescentes" e "todo o grupo", respectivamente, sendo para o masculino uma prevalência de 55,4, 74,8 e $69,8 \%$ para os mesmos grupos considerados. Não houve diferenças quando consideradas as hTV entre os sexos e nem ao comparar escolares sedentários com ativos fisicamente $(p>0,05)$. Concluiu-se que: a) há alta prevalência de crianças e adolescentes caracterizados como "sedentários" e "muito sedentários"; b) o sexo masculino apresentou maiores NAF que o feminino c) adolescentes são menos ativos do que as crianças. d) há grande quantitativo de hTV no grupo estudado.

Palavras-chave: Atividade motora; Estudantes; Estudos transversais.

1 Universidade Federal de Santa Catarina, Núcleo de Pesquisa em Cineantropometria e Desempe nho Humano. Florianópolis, SC. Brasil.

2 Universidade Tiradentes, Grupo de Pesquisa em Atividade Física Relacionada à Saúde. Aracaju, SE. Brasil.

Recebido em 18/12/07 Aprovado em 24/07/08
Abstract - The objective of this study was to determine the physical activity level (PAL) and sedentary behavior of students from the city of Aracaju (SE). A total of 1028 students of both genders participated in the study, with a mean age of 15.38 (2.44) years for girls and 15.24 (2.40) years for boys. Among the sample, $24.7 \%$ were children and $75.3 \%$ were adolescents, with a mean age of 12.07 (0.88) and 16.39 (1.72) years, respectively. An instrument already used in Brazilian studies was applied to identify the average time (hours) watching TV per day (hTV) and PAL - PAQ-C. Descriptive statistics, t-test for independent samples, Fisher's exact test and comparison test between two proportions were used for data analysis, with the level of significance set at $5 \%(p \leq 0.05)$. Boys presented a significantly higher physical activity score $(2.25(0.60))$ than girls. The prevalence of sedentarism was $72.5,89.3$ and $85.2 \%$ in the groups of children and adolescents and in the group as a whole, respectively, for girls, and 55.4, 74.8 and 69.8\% for boys. No differences in $h T V$ were observed between genders or between sedentary and physically active students ( $p>0.05)$. We conclude that a) there is a high prevalence of "sedentary" and "very sedentary" children and adolescents; b) boys present a higher PAL than girls; c) adolescents are less active than children, and d) the number of hTV is high in the group studied. Key words: Motor activity; Students; Cross-sectional study. 


\section{INTRODUÇÃO}

O nível de atividade física e a prevalência de sedentarismo são estudados em vários grupos como crianças $^{1-3}$, adolescentes ${ }^{3-5}$, adultos ${ }^{6}$, idosos ${ }^{7}$ e portadores de problemas crônico-degenerativos ${ }^{8}$. As crianças e adolescentes têm obtido uma atenção especial, pois é nesta fase da vida que os hábitos e os comportamentos são formados, e refletem para a idade adulta ${ }^{5,9-10}$.

Alguns estudos demonstram que existe alta prevalência de comportamentos de risco à saúde em crianças e adolescentes ${ }^{11-13}$, dentre estes comportamentos, destacam-se os níveis insuficientes de atividade física, hábitos alimentares inadequados, consumo de drogas lícitas e ilícitas e comportamento sexual inseguro.

Twisk ${ }^{4}$ enfatiza que a prática de atividade física pelos jovens, provavelmente, torná-los-ão adultos fisicamente ativos, e uma das formas de promover este estilo de vida em crianças e adolescentes é por meio de programas escolares de educação física. Estes programas têm que objetivar a adoção de hábitos saudáveis, tanto no ambiente escolar, quanto nas horas de lazer ${ }^{10,14}$.

Investigações demonstram que crianças e adolescentes tem um grande componente de tempo livre não estruturado, e que cerca de $40 \%$ deste poderia ser revertido em atividades físicas ${ }^{9}$. Contudo, a maioria das atividades que os jovens fazem é de característica sedentária, como assistir TV e participar de jogos eletrônicos ${ }^{9,15}$. Esta crescente forma de lazer sedentário dos jovens das grandes cidades foi reportada por Silva e Malina ${ }^{16}$ como um meio barato e seguro de lazer, que favorece o aumento do quantitativo de horas de assistência à TV e comportamentos sedentários.

Pesquisas em saúde pública ressaltaram que ambientes onde os jovens passam boa parte do dia podem servir como incentivadores de um estilo de vida mais saudável e ativo ${ }^{15,17-18}$. Neste sentido, a escola é um ambiente fundamental para adoção destes comportamentos e evidências de efetividade de programas de intervenção aqui no Brasil já foram reportadas ${ }^{13}$, o que serve como incentivadores de outros programas nestes locais. Contudo, para a realização de programas desta natureza, faz-se necessário o levantamento prévio dos níveis de atividade física e comportamentos de risco da população.

Os poucos estudos que descreveram o nível de atividade física e alguns comportamentos entre os escolares de capitais da região Nordeste do Brasil foram reportados, recentemente, em uma revisão sistemática ${ }^{19}$. A partir desta revisão infere-se que investigações sobre o nível de atividade física de jovens das regiões brasileiras são necessárias, bem como a associação destes níveis com comportamentos sedentários. Pois ainda não está evidente se jovens com maiores horas de assistência à TV tendem a apresentar menores níveis de atividade física, tendo em vista que a depender da localidade, isto ocorre ${ }^{17}$ ou não ${ }^{16}$.

Assim, o presente trabalho tem como objetivo verificar o nível de atividade física e o comportamento sedentário em crianças e adolescentes escolares da cidade de Aracaju (SE).

\section{PROCEDIMENTOS METODOLÓGICOS}

\section{População e Amostra}

Este estudo de delineamento transversal, de base escolar, é parte do projeto intitulado Crescimento, composição corporal e desempenho físico em crianças e adolescentes do município de Aracaju (SE), aprovado pelo Comitê de Ética da Universidade Tiradentes, sob número 12/04, com financiamento do CNPq/MS/SES/FAPITEC.

A população deste estudo foi composta por estudantes do ensino fundamental e médio da Rede Pública Estadual de Ensino do município de Aracaju. Para o levantamento de informações, este município foi dividido em quatro regiões de acordo com a organização da cidade: Norte, Sul, Oeste e Centro.

Para a caracterização amostral, utilizou-se a maior escola de cada região da cidade em quantitativo de alunos. Pois as mesmas continham alunos de todos os pontos de cada região. Para a definição da representatividade amostral, tomaram-se por referência as informações sobre as Unidades Escolares apresentadas pela Secretaria de Estado da Educação. Dessa forma, foi obtida uma população de 5804 alunos.

Para a definição do quantitativo de sujeitos participantes do estudo, adotou-se um nível de confiança de 1,96, com um erro amostral de 3\%. Para minimizar a possibilidade de perda amostral, o valor mínimo calculado foi acrescido em $10 \%$, o que resultou no mínimo necessário de 1026 estudantes.

Os alunos foram definidos por conglomerado de série escolar. Os critérios de exclusão foram: não entrega do Termo de Consentimento Livre e Esclarecido assinado pelos pais ou responsáveis e o não interesse do aluno em participar da coleta dos dados.

Desta maneira, o presente estudo foi realizado com 1028 crianças e adolescentes das quatro escolas públicas estaduais escolhidas, consideradas as 
regiões do município. $\mathrm{Na}$ Tabela 1 , encontra-se o quantitativo amostral sobre as informações demográficas dos escolares do presente estudo.

Tabela 1. Quantitativo amostral por variáveis demográficas dos escolares de Aracaju.

\begin{tabular}{lcc}
\hline Variáveis demográficas & $\mathrm{n}$ & $\%$ \\
\hline Sexo & & \\
$\quad$ Feminino & 583 & 56,7 \\
$\quad$ Masculino & 445 & 43,3 \\
Grupo etário & & \\
$\quad$ Criança & 254 & 24,7 \\
$\quad$ Adolescente & 774 & 75,3 \\
\hline Região geográfica & & \\
\hline$\quad$ Norte & 290 & 28,2 \\
Sul & 368 & 35,8 \\
Oeste & 237 & 23,1 \\
Centro & 133 & 12,9 \\
\hline
\end{tabular}

Dentre os sujeitos que participaram do estudo, $445(43,3 \%)$ eram do sexo masculino, com uma média de 15,25 (desvio padrão - dp - 2,40) anos de idade e, $583(56,7 \%)$ do sexo feminino, com uma média de 15,38 (dp - 2,44) anos de idade.

Para verificar possíveis diferenças nos comportamentos propostos do presente estudo entre os escolares mais jovens e os mais velhos, decidiu-se dicotomizar a amostra em relação à faixa etária. Assim, sujeitos menores de 13 anos e com idade igual ou superior a 13 anos formaram os dois grupos. Para melhor visualização destas categorias, o grupo com idade inferior a 13 anos foi denominado de "crianças" $(\mathrm{n}=254)$ e, o outro grupo de "adolescentes" (n=774). No presente estudo, não foram analisadas diferenças interindividuais e nem entre os sexos em relação ao estatuto maturacional para a caracterização de crianças e adolescentes, desta feita, a divisão considerada foi meramente didática. O sexo feminino apresentou uma média de $12,11(\mathrm{dp}-0,86)$ e $16,43(\mathrm{dp}-1,77)$ anos de idade, respectivamente, para crianças e adolescentes e, o masculino de $12,02(\mathrm{dp}-0,90)$ e $16,33(\mathrm{dp}-1,66)$ anos de idade, respectivamente.

\section{Instrumentos e Procedimentos}

A coleta de dados foi desenvolvida a partir de um instrumento já utilizado no Brasi ${ }^{16}$, com perguntas relacionadas ao nível de atividade física (NAF) e tempo de assistência à TV.

Foi utilizado o questionário $\mathrm{PAQ}-\mathrm{C}^{20}$, o qual contém perguntas que investigam o NAF de crianças e adolescentes nos sete dias anteriores ao preenchimento do questionário. Neste instrumento, cada questão tem valor de 1 a 5 e o escore final é obtido pela média das questões, que representa o intervalo de "muito sedentário" (1) a "muito ativo" (5). Os escores 2, 3 e 4 indicam as categorias "sedentário", "moderadamente ativo" e "ativo", respectivamente. Além destas classificações provenientes do questionário, o presente estudo também utilizou uma dicotomização de tais situações: "inadequada" (muito sedentário + sedentário) e "adequada" (ativo + muito ativo) para saúde.

Para verificar o tempo de assistência à TV dos escolares, utilizou-se uma pergunta do questionário $\mathrm{PAQ}-\mathrm{C}^{20}$ que não entra no cômputo do escore de atividade física.

\section{Tratamento estatístico}

Os procedimentos utilizados para a análise de dados foram a estatística descritiva para apresentação das informações encontradas (média, desvio padrão, freqüência absoluta e relativa); o teste t para amostras independentes quando as comparações foram entre os sexos; e o teste Exato de Fisher para verificar a associação entre as variáveis NAF e sexo. Para identificar possíveis diferenças entre as proporções de cada categoria do NAF entre os sexos e entre sedentários e ativos para o grupo das crianças, adolescentes e ao considerar toda a amostra, foi empregado o teste de comparação entre duas proporções. Em todas as análises adotou-se um nível de significância de 5\% (p $\leq 0,05)$.

\section{RESULTADOS}

A Tabela 2 apresenta a caracterização do grupo estudado, assim como os valores obtidos para a comparação entre os sexos, no que se refere aos escores obtidos a partir da análise do NAF e ao quantitativo de horas assistindo TV.

No escore do NAF, tanto para crianças quanto para os adolescentes, houve uma diferença significativa entre os sexos, favorável ao masculino. Quando considerado todo o grupo, a diferença permanece $(\mathrm{p}<0,05)$.

Em relação ao tempo médio de horas de assistência à TV não foram encontradas diferenças significativas entre os sexos, tanto para o subgrupo de crianças quanto para os adolescentes $(\mathrm{p}>0,05)$.

Na Tabela 3, verificam-se as proporções de escolares classificados quanto ao NAF em relação ao sexo. Foi observada associação significativa entre as categorias do NAF e o sexo $(\mathrm{p}<0,05)$. Ao utilizar o teste de comparação entre duas proporções para identificar em quais categorias foram as diferenças, observou-se que nas crianças, nos adolescentes e, ao 
Tabela 2. Valores médios e o desvio padrão do escore do nível de atividade física e das horas assistindo TV de escolares de Aracaju, SE.

\begin{tabular}{|c|c|c|c|}
\hline \multirow{2}{*}{ Variável } & \multicolumn{2}{|c|}{ Sexo } & \multirow{2}{*}{$\mathrm{p}$} \\
\hline & Feminino & Masculino & \\
\hline \multicolumn{4}{|l|}{ Crianças } \\
\hline $\mathrm{n}(\%)$ & $142(55,91)$ & $112(44,09)$ & - \\
\hline Escore do NAF & $2,23(0,57)$ & $2,49(0,59)$ & $<0,001^{*}$ \\
\hline Horas assistindo TV & $6,31(4,71)$ & $6,35(3,40)$ & 0,940 \\
\hline \multicolumn{4}{|l|}{ Adolescentes } \\
\hline $\mathrm{n}(\%)$ & $441(56,98)$ & $333(43,02)$ & - \\
\hline Escore do NAF & $1,86(0,58)$ & $2,10(0,59)$ & $<0,001^{*}$ \\
\hline Horas assistindo TV & $5,50(3,96)$ & $5,25(3,35)$ & 0,350 \\
\hline \multicolumn{4}{|l|}{ Todo o Grupo } \\
\hline $\mathrm{n}(\%)$ & $583(56,7)$ & $445(43,3)$ & - \\
\hline Escore do NAF & $1,95(0,60)$ & $2,25(0,60)$ & $<0,001^{*}$ \\
\hline Horas assistindo TV & $5,70(4,16)$ & $5,50(3,54)$ & 0,460 \\
\hline
\end{tabular}

$\mathrm{NAF}$ - nível de atividade física; *Diferença significativa entre os sexos - teste " $\mathrm{t}$ " para amostras independentes ( $\mathrm{p}<0,05)$.

Tabela 3. Proporção de escolares por sexo quanto à classificação do nível de atividade física.

\begin{tabular}{|c|c|c|c|}
\hline \multirow[b]{2}{*}{ NAF } & \multicolumn{2}{|c|}{ Sexo } & \multirow[b]{2}{*}{$\mathbf{p}$} \\
\hline & $\begin{array}{l}\text { Feminino } \\
\mathrm{n}(\%)\end{array}$ & $\begin{array}{c}\text { Masculino } \\
\mathrm{n}(\%)\end{array}$ & \\
\hline Crianças & & & $0,002^{*}$ \\
\hline Muito sedentário ${ }^{+}$ & $8(5,6)$ & $0(0)$ & \\
\hline Sedentário & $95(66,9)$ & $62(55,4)$ & \\
\hline Moderadamente Ativo ${ }^{+}$ & $37(26,1)$ & $45(40,2)$ & \\
\hline Ativo & $2(1,4)$ & $5(4,5)$ & \\
\hline Total & $142(100)$ & $112(100)$ & \\
\hline Adolescentes & & & $0,000^{*}$ \\
\hline Muito sedentário $^{+}$ & $110(24,9)$ & $30(9,0)$ & \\
\hline Sedentário & $284(64,4)$ & $219(65,8)$ & \\
\hline Moderadamente Ativo ${ }^{+}$ & $47(10,7)$ & $80(24,0)$ & \\
\hline Ativo & $0(0)$ & $4(1,2)$ & \\
\hline Total & $441(100)$ & $333(100)$ & \\
\hline Todo o Grupo & & & $0,000^{*}$ \\
\hline Muito sedentário ${ }^{+}$ & $118(20,2)$ & $30(6,7)$ & \\
\hline Sedentário & $379(65,0)$ & $281(63,1)$ & \\
\hline Moderadamente Ativo $^{+}$ & $84(14,4)$ & $125(28,1)$ & \\
\hline Ativo $^{+}$ & $2(0,3)$ & $9(2)$ & \\
\hline Total & $583(100)$ & $445(100)$ & \\
\hline
\end{tabular}

NAF - nível de atividade física; * Associação entre NAF e sexo - Teste Exato de Fisher ( $<<0,05)$; † Diferença entre as proporções comparando os sexos - teste de comparação entre duas proporções $(p<0,05)$

considerar todo o grupo, a prevalência de sujeitos muito sedentários foi maior estatisticamente no sexo feminino. A prevalência de indivíduos moderadamente ativos foi maior estatisticamente no sexo masculino. Já ao considerar todo o grupo, a proporção de escolares do sexo masculino classificado como ativo fisicamente é maior estatisticamente do que o feminino $(p<0,05)$.

Em relação ao comportamento sedentário analisado, observa-se, na tabela 4, o tempo médio despendido em frente da TV. Foi identificado que as crianças do sexo masculino classificadas com NAF inadequado passavam mais horas do dia em frente da TV do que as com NAF adequado $(p=0,041)$, entretanto, nas crianças do sexo feminino foi identificado comportamento contrário $(p=0,034)$. Para os adolescentes e para todo o grupo, não foram encontradas diferenças significativas no tempo de assistência à TV entre os escolares com NAF inadequado e adequado ( $p>0,05)$. 
Tabela 4. Valores médios e o desvio padrão das horas por dia em frente da TV.

\begin{tabular}{lccc}
\hline & \multicolumn{2}{c}{ Horas de assistência à TV } & NAF \\
\cline { 2 - 4 } Variável & NAF & & \\
Inadequado & & $7,29(4,55)$ & $0,041^{*}$ \\
\hline Crianças & $5,60(3,37)$ & $5,17(3,02)$ & $0,034^{*}$ \\
Masculino & $6,74(5,16)$ & & 0,175 \\
Feminino & & $4,81(2,96)$ & 0,941 \\
Adolescentes & $5,40(3,47)$ & $5,55(3,93)$ & \\
Masculino & $5,50(3,97)$ & $5,66(3,76)$ & 0,579 \\
Feminino & & $5,38(3,53)$ & 0,397 \\
Mado o Grupo & $5,44(3,45)$ & $5,75(4,26)$ & \\
\hline
\end{tabular}

NAF - Nível de atividade física; * $\mathrm{p}<0,05$ (teste " $\mathrm{t}$ " para amostras independentes)

\section{DISCUSSÃO}

Ao verificar os escores do NAF das crianças, ou somente dos adolescentes, ficou evidenciado que os escolares do sexo masculino são mais ativos fisicamente do que os do sexo feminino. Resultados semelhantes foram reportados em estudos internacionais ${ }^{20-21}$ e nacionais ${ }^{16}$ que utilizaram o mesmo instrumento de medida da atual investigação. Estudos que utilizaram outros instrumentos de medida, seja de forma direta ou indireta, também encontraram que o sexo masculino é mais ativo fisicamente do que o feminino ${ }^{17,22-23}$. Pesquisadores reportam que estas diferenças na adoção de um hábito ou prática podem ser devido a fatores culturais, comportamentais, psicológicos e, até mesmo, maturacionais que afetam esta faixa etária藏, o que reflete de forma diferente entre os sexos. O presente estudo não considerou os diferentes fatores que podem influenciar um comportamento, portanto, futuras investigações que considerem todos os fatores citados são recomendadas na população estudada.

A prevalência de indivíduos classificados como sedentários (muito sedentários e sedentários) foi de $78,6 \%$, sendo $64,9 \%$ de crianças e $83,1 \%$ de adolescentes. Estas prevalências estão em conformidade com o estudo de revisão sistemática realizado no Brasil para identificar a prevalência de sedentarismo em jovens, que reportou uma variação de 39\% a 93,5\% de sedentarismo ${ }^{19}$. A prevalência encontrada no presente estudo pode ser considerada alta devido aos diversos prejuízos à saúde causados pelo sedentarismo, como diabetes, obesidade, hipertensão arterial, coronariopatias, alguns tipos de câncer e osteoporose $^{24}$. Por isso, um alerta aos setores da educação e saúde do município investigado faz-se necessário para a implantação de políticas e programas de combate ao sedentarismo e promoção da atividade física.
A escassez de estudos que investigaram o NAF em crianças e adolescentes da região Nordeste é evidente, o que dificulta a comparação com os dados do presente estudo. Dentre as poucas investigações, destaca-se uma realizada com escolares de 7 a 17 anos de idade da cidade de Maceió-AL, que reportou sedentarismo em 93,5\% dos estudantes ${ }^{25}$. Magalhães et al. ${ }^{26}$ ao verificarem a prevalência e os fatores associados ao sobrepeso e obesidade em adolescentes de 15 a 19 anos, das regiões Nordeste e Sudeste do Brasil, encontraram que $40,7 \%$ do sexo masculino e $80,0 \%$ do feminino reportaram não realizar nenhuma atividade física de lazer por semana.

No Brasil, para a estimativa de sedentarismo em jovens, não existe uma padronização de instrumentos de medidas de atividade física, seja ele direto ou indireto. Nos diferentes estudos já realizados, a maior parte fez uso de um instrumento diferente ${ }^{16,27-28}$, o que prejudica as comparações entre as pesquisas e o conhecimento dos reais níveis de atividade física dos jovens brasileiros. Esta não padronização dificulta possíveis intervenções de combate ao sedentarismo e promoção da atividade física no ambiente escolar, pois os diferentes instrumentos foram criados embasados em investigações realizadas em países desenvolvidos, com condições sociais, econômicas e culturais diferentes do Brasil. Portanto, é necessário que a adequação ou a criação de um instrumento de medida baseado nas condições brasileiras seja elaborado para um melhor entendimento dos padrões de atividade física dos jovens.

$\mathrm{Na}$ atual pesquisa, pôde-se verificar que, a prevalência de escolares muito sedentários e sedentários foi maior nos adolescentes do que nas crianças. Resultados semelhantes foram reportados em investigações transversais nacionais ${ }^{25}$, internacionais ${ }^{17} \mathrm{e}$ em estudos longitudinais ${ }^{21,23}$ que 
demonstraram uma queda no NAF com o passar da idade. Além de baixos níveis de atividade física, a literatura também evidencia a adoção de outros comportamentos de risco à saúde com o passar da idade, como por exemplo, baixo consumo de frutas e verduras, consumo de tabaco e álcool e comportamento sexual inseguro $^{29}$. Desta feita, a investigação sobre NAF em escolares é uma das partes para o entendimento do estilo de vida dos jovens. Pesquisas adicionais que considerem os diversos comportamentos de saúde são aqui recomendadas para intervenções de promoção da saúde mais abrangentes no meio escolar.

Em relação a horas de assistência à TV, a média de tempo encontrada no presente estudo foi de $5,61(\mathrm{dp}-3,85)$ horas por dia. Este quantitativo é preocupante, pois pesquisas com jovens em outras cidades brasileiras verificaram um menor tempo de assistência à $\mathrm{TV}^{12,16,28} \mathrm{e}$, naqueles estudos as horas despendidas à frente da televisão mostrou-se associada a diversos fatores de risco, como o elevado percentual de gordura ${ }^{12}$ e sobrepeso ${ }^{18}$.

Nitzan Kaluski et al. ${ }^{17}$ indicaram que o sexo feminino passa mais tempo assistindo TV do que o masculino e, talvez por isso, pudesse apresentar menores níveis de atividade física. Contudo, estas considerações não serviram para o presente estudo, pois como observado na Tabela 2, os escolares do sexo feminino apresentaram uma média de horas ao dia assistindo TV de 5,70 (dp - 4,16) e o masculino de 5,50 (dp - 3,54), não existindo diferenças significativas entre os sexos. Pesquisa realizada em Niterói-RJ encontrou resultado semelhante ao do presente estudo ${ }^{16}$.

Ao comparar, na Tabela 4, as horas médias por dia que os escolares assistem TV, foi encontrado um resultado interessante para as crianças. Pois as crianças do sexo masculino com NAF adequado passaram mais tempo vendo TV do que os de NAF inadequado. Este resultado não era esperado, pois outros autores reportam relação inversa entre comportamento sedentário e $\mathrm{NAF}^{17}$, bem como foi verificado ao comparar as crianças Aracajuanas do sexo feminino. Para os adolescentes e para todo o grupo de escolares do presente estudo, não foram encontradas diferenças significativas no tempo de assistência à TV entre aqueles de NAF adequado e inadequado. Desta maneira, infere-se que o comportamento sedentário de assistência à TV não influenciou os níveis de atividade física de escolares Aracajuanos, e estes baixos níveis de atividade física encontrados são devido a outros fatores comportamentais que necessitam ser investigados.
O tempo de assistência à TV por parte de crianças e adolescentes e os demais comportamentos sedentários não investigados no presente estudo como, tempo à frente do computador e do vídeo game, são condutas que necessitam de investigações detalhadas. Tais comportamentos se associam à ingestão de comidas com muitas calorias, pobre em nutrientes e ricas em gorduras, o que prejudica a saúde ${ }^{30}$. Neste sentido, futuras investigações que considerem tempo de assistência à $\mathrm{TV}$, atividade física, hábitos alimentares e outros indicadores do estilo de vida são necessários para maiores inferências entre os escolares de Aracaju.

Algumas limitações da presente investigação podem ser citadas, como o fato de ser um estudo transversal, o que impossibilita a verificação da relação de causalidade entre as variáveis investigadas. No entanto, estudos transversais são importantes para a identificação de problemas e elaboração de hipóteses para pesquisas prospectivas. Além desta limitação, têm-se o fato do estudo ser de base escolar, o que dificulta a generalização para outros locais brasileiros e para jovens que não frequentam a escola ou que não a frequentavam no período da coleta dos dados.

O presente estudo apresenta uma contribuição para a área, pois trouxe dados referentes ao NAF e ao comportamento sedentário de crianças e adolescentes da cidade de Aracaju, sendo uma das poucas investigações desta natureza realizada com amostra representativa de escolares de uma capital da região nordeste do Brasil. Este fato pode servir de parâmetro comparativo para próximas investigações realizadas em outras cidades da região.

\section{CONCLUSÃO}

Ao considerar os resultados do presente estudo, pode-se concluir que em relação ao NAF os resultados estão de acordo com as estimativas encontradas nas diferentes cidades brasileiras, com a prevalência de sedentários maior do que a de indivíduos ativos. Além disso, o sexo feminino e os adolescentes foram os grupos com maiores prevalências de sedentarismo. A respeito do quantitativo de horas gastas pelos escolares em assistência à TV, este comportamento foi semelhante entre os sexos e, em média, os jovens de Aracaju passam mais tempo à frente da TV do que o de outras cidades brasileiras. Ademais, o NAF dos escolares de Aracaju não sofreu influência do tempo de assistência à TV.

Estas conclusões sugerem uma ação efetiva quanto à orientação das crianças e adolescentes 
para a adoção de um estilo de vida ativo e reforça a importância do ambiente escolar nesta mudança de comportamento, sobretudo, por ser o local onde os jovens passam boa parte do dia e, consequentemente, podem aprender e adotar comportamentos mais adequados para toda vida.

\section{REFERÊNCIAS BIBLIOGRÁFICAS}

1. Kelishadi R, Ardalan G, Gheiratmand R, Gouya MM, Razaghi EM, Delavari A, et al. Association of physical activity and dietary behaviours in relation to the body mass index in a national sample of Iranian children and adolescents: CASPIAN Study. Bulletin of World Health Organization 2007;85(1):19-26.

2. Armstrong N, Welsman JR. The physical activity patterns of European youth with reference to methods of assessment. Sports Med 2006;36(12):1067-1086.

3. Gustafson SL, Rhodes RE. Parental correlates of physical activity in children and early adolescents. Sports Med 2005;36(1):79-97.

4. Twisk JWR. Physical activity guidelines for children and adolescents: A critical review. Sports Med 2001;3(8):617-627.

5. Torres LAP, Lluch C, Moral RR, Espejo JE, Tapia G, Luque PM. Prevalencia de actividad fisica y su relación con variables sociodemográficas y ciertos estilos de vida en escolares cordobenses. Rev Esp de Salud Publica 1998;72 (3):233-244.

6. Van Mechelen W, Twisk JWR, Post G. Bertheke et al. Physical activity of young people: the Amsterdam Longitudinal Growth and Health Study. Med Sci Sports Exerc 2000;32(9):1610-1616.

7. Benedetti TR, Borges LJ, Petroski EL, Gonçalves LH. Atividade física e estado de saúde mental de idosos. Rev Saude Publica 2008;42(2):302-307.

8. Hu G, Jousilahti P, Antikainen R, Tuomilehto J. Occupational, commuting, and leisure-time physical activity in relation to cardiovascular mortality among finnish subjects with hypertension. Am J Hypertens 2007;20(12):1242-1250.

9. Esculcas C, Mota J. Actividade física e práticas de lazer em adolescentes. Rev Port Ciências Desp 2005;5(1):69-76.

10. Tudor-Locke C, Ainsworth BE, Popkin BM. Active commuting to school: an overlooked source of childrens physical activity? Sports Med 2001;31(5):309-313.

11. Dietz WH. Overweight in childhood and adolescence. Perspective 2004;9(350):855-857.

12. Pelegrini A, Silva RCR, Petroski EL. Relação entre o tempo em frente à TV e o gasto calórico em adolescentes com diferentes percentuais de gordura corporal. Rev Bras Cineantropom Desempenho Humano 2008;10(1):81-84.

13. Barros MVG, Nahas MV, Hallal PC, Farias Junior JC, Florindo AA, Barros SSH. Effectiveness of a School-
Based Intervention on Physical Activity for High School Students in Brazil: The Saude na Boa Project. J Phys Act Health 2008;6(2):163-169.

14. Trudeau F, Shephard RJ. Contribution of school programmes to physical activity levels and attitudes in children and adults. Sports Med 2005;35(2):89-105.

15. Moraes SA, Rosas JB, Mondini L, Freitas ICM. Prevalência de sobrepeso e obesidade e fatores associados em escolares de área urbana de Chilpancingo, Guerrero, México, 2004. Cad Saúde Pública 2006;22(6):1289-1301.

16. Silva RCR, Malina RM. Nível de atividade física em adolescentes do Município de Niterói, Rio de Janeiro, Brasil. Cad Saúde Pública 2000;16 (4):1091-1097.

17. Nitzan Kaluski D, Demem Mazengia G, Shimony T, Goldsmith R, Berry EM. Prevalence and determinants of physical activity and lifestyle in relation to obesity among schoolchildren in Israel. Public Health Nutr 2009 (in press).

18. Kaur H, Choi WS, Mayo MS, Jo HK. Duration of television watching is associated with increased body mass index. J Pediatr 2003;143(4):506-511.

19. Tassitano RM, Bezerra J, Tenório MCM, Colares V, Barros MVG, Hallal PC. Atividade física em adolescentes brasileiros: uma revisão sistemática. Rev Bras Cineantropom Desempenho Hum 2007;9(1):55-60.

20. Crocker PR, Bailey DA, Faulkner RA, Kowalski KC, McGrath R.. Measuring general levels of physical activity: Preliminary evidence for the Physical Activity Questionnaire for Older Children. Med Sci Sports Exerc 1997;29 (10):1344-1349.

21. Thompson AM, Baxter-Jones AD, Mirwald RL, Bailey DA. Comparison of physical activity in male and female children: does maturation matter? Med Sci Sports Exerc 2003;35(10):1684-1690.

22. Duncan MJ, Woodfield L, Al-Nakeeb Y, Nevill AM. Differences in physical activity levels between white and South asian children in the United kingdom. Pediatr Exerc Sci 2008;20(3):285-291.

23. Nader PR, Bradley RH, Houts RM, McRitchie SL, O'Brien M. Moderate-to-vigorous physical activity from ages 9 to 15 years. JAMA 2008;300(3):295-305.

24. Haskell WL, Lee IM, Pate RR, Powell KE, Blair SN, Franklin BA, et al. American College of Sports Medicine; American Heart Association. Physical activity and public health: updated recommendation for adults from the American College of Sports Medicine and the American Heart Association. Circulation 2007;116(9):1081-1093.

25. Da Silva M, Rivera I, Ferraz M, Pinheiro A, Alves Moura A, Carvalho ACC. Prevalência de fatores de risco cardiovascular em crianças e adolescentes da rede de ensino da cidade de Maceió. Arq Bras Cardiol 2005;84(5):387-392.

26. Magalhães V, Mendonça S. Prevalência e fatores associados a sobrepeso e obesidade em adolescentes de 15 a 
19 anos das regiões Nordeste e Sudeste do Brasil, 1996 a 1997. Cad Saúde Pública 2003;19:S129-139.

27. Pinho RA, Petroski EL. Nível habitual de atividade física e equilíbrio energético de adolescentes. Rev Bras Ativ Fís Saúde 1999;4 (2):5-16.

28. Hallal PC, Bertoldi AD, Gonçalves H, Victora CG. Prevalência de sedentarismo e fatores associados em adolescentes de 10-12 anos de idade. Cad. Saúde Pública 2006;22(6):1277-1287.

29. Steptoe A, Wardle J, Cui W, Bellisle F, Zotti A, Baranyai R, et al. Trends in smoking, diet, physical exercise, and attitudes toward health in European university students from 13 countries, 1990-2000. Prev Med 2002;35 (2):97-104.
30. Blass EM, Anderson DR, Kirkorian HL, Pempek TA, Price I, Koleini MF. On the road to obesity: Television viewing increases intake of high-density foods. Physiol Behav 2006;30(88):597-604.

\section{Endereço para correspondência}

Roberto Jerônimo dos Santos Silva

Rua Professor Jugurta Feitosa Franco, 334

Condomínio Residencial San Francisco, 104 C

Bairro Coroa do Meio

49035-690 - Aracaju, SE. Brasil

E-mail: rjeronimoss@gmail.com 